\title{
COMPUTATIONAL CHEMISTRY
}

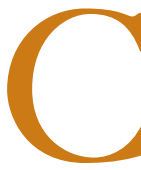

omputational chemistry has come of age. With significant strides in computer hardware and software over the last few decades, computational chemistry has achieved full partnership with theory and experiment as a tool for understanding and predicting the behavior of a broad range of

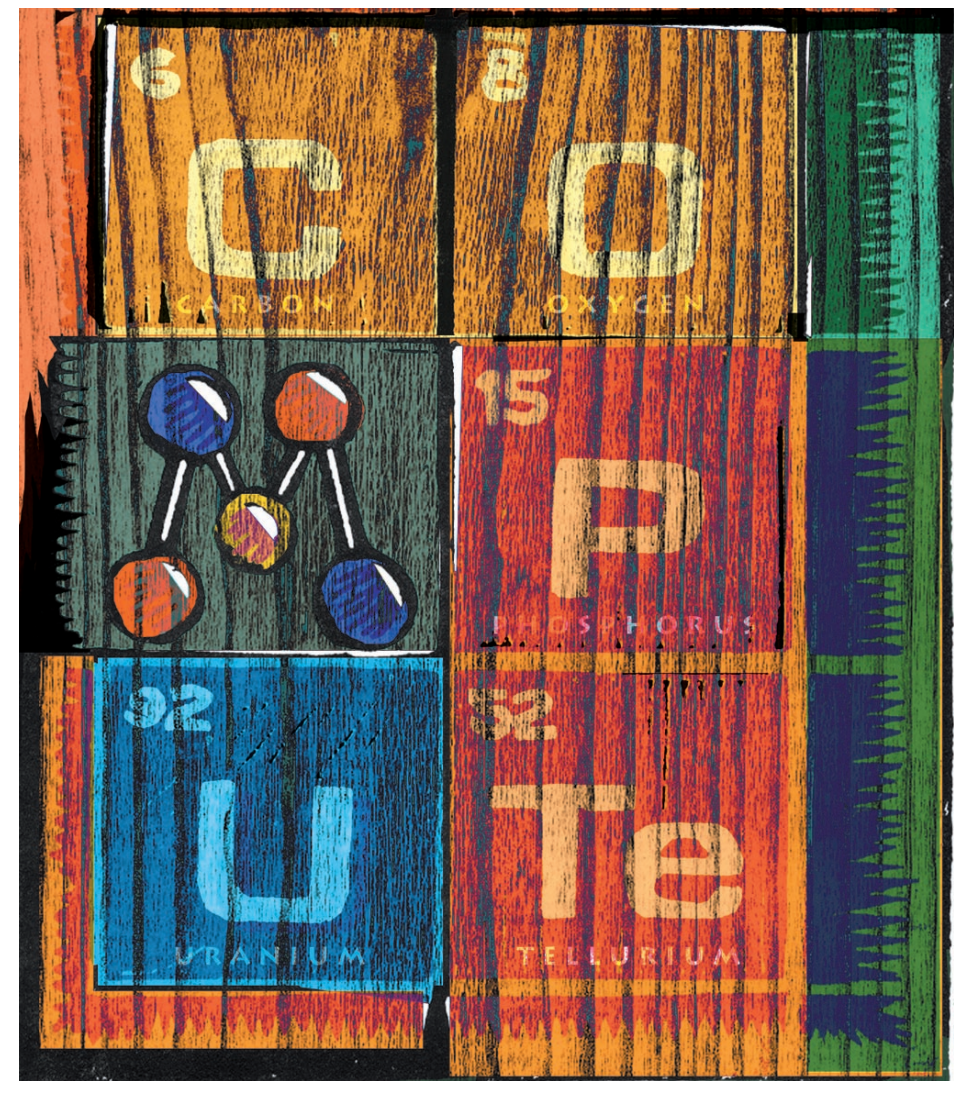

$1521-9615 / 00 / \$ 10.00 \odot 2000$ IEEE

Donald G. TRUhlaR

University of Minnesota

VINCENT MCKOY

California Institute of Technology chemical, physical, and biological phenomena. The Nobel Prize award to John Pople and Walter Kohn in 1998 highlighted the importance of these advances in computational chemistry. With massively parallel computers capable of peak performance of several teraflops already on the scene and with the development of parallel software for efficient exploitation of these highend computers, we can anticipate that computational chemistry will continue to change the scientific landscape throughout the coming century. The impact of these advances will be broad and encompassing, because chemistry is so central to the myriad of advances we anticipate in areas such as materials design, biological sciences, and chemical manufacturing.

\section{Application areas}

Materials design is very broad in scope. It addresses a diverse range of compositions of matter that can better serve structural and functional needs in all walks of life. Catalytic design is one such example that clearly illustrates the promise and challenges of computational chemistry. Almost all chemical reactions of industrial or biochemical significance are catalytic. Yet, catalysis is still more of an art (at best) or an empirical fact than a design science. But this is sure to change.

A recent American Chemical Society Symposium volume on transition state modeling in catalysis illustrates its progress. ${ }^{1}$ This symposium highlighted a significant development in the level of modeling that researchers are employing as they focus more and more on the highly quantum mechanical activated complexes of catalytic reactions. The complexity of catalytic processes had previously restricted fullscale computational efforts to the structure and binding of the reactive precursors. The impli- 
cations of this shift will be profound but not immediate. A relevant comparison is computeraided drug design - it became clear some 20 years ago that the computer-aided approach held tremendous promise. Although there has been impressive progress in the area, ${ }^{2,3}$ the majority of successful drugs currently on the market were developed without the aid of computers or on the basis of rudimentary computational techniques that are no longer state of the art. However, this is changing ever more rapidly.

A major challenge to materials design, as well as to many other areas of chemical modeling such as the environment, is the wide range of length and time scales that we must address. ${ }^{4}$ The operative objective is nanoscale modeling. For example, a cubic sample $5 \mathrm{~nm} \times 5 \mathrm{~nm} \times 5$ $\mathrm{nm}$ on edge contains thousands of atoms, and a simulation over 5 ns encompasses $10^{5}$ vibrational periods of a strong bond to a hydrogen atom. Following the motion of such a large number of atoms for a long time is challenging, but computational chemists are not waiting for computers to get faster. They are devising algorithms specifically designed to address these issues. An example is the hyperdynamics algorithm ${ }^{5}$ of Art Voter at Los Alamos National Laboratory, which "floods the valleys" and lifts every molecule like a ship (so to speak) so it can more easily reach the high-energy dynamical bottlenecks. The algorithm then carefully corrects for the artificial bias so that we can predict observables in real time.

Improving manufacturing processes to make them more environmentally benign and to rationally design new materials will increasingly rely on computational chemistry. The rapid and efficient design of new materials, chemical intermediates, and products will be necessary to achieve the goals of greater energy efficiency and increased productivity while minimizing environmental impact.

Combustion is another area where the computational approach really shines. A better understanding of the underlying processes in combustion can have significant impact on our utilization of energy resources. A typical combustion system involves hundreds of unstable chemical intermediates, most of which researchers have never isolated for individual experimental study. Theory can predict their heats of formation and their subsequent reaction fates almost as easily as we can calculate the properties of the stable molecules for which we have measured these attributes. The value of such calculations for the design of cleaner, more efficient combustion processes should be obvious.

\section{Theme issue}

This theme issue cannot possibly cover all the excitement of the computational chemistry field, but the articles presented here provide a varied picture of four exciting research areas.

"Simulating Complex Systems without Adjustable Parameters" by Michele Parrinello looks at the progress, computational challenges, and future potential of molecular dynamics. The potential applications of molecular dynamics are almost unlimited. The fascination and usefulness of this approach stem from its ability to provide a window of increasing spatial and temporal resolution on the behavior of complex systems.

In "Atomic Scale Modeling of Polymerization Catalysts," Tom Woo, Serguei Patchkovskii, and Tom Ziegler discuss an application of molecular modeling to examine a catalytic cycle's elementary reaction steps at the atomic level. They also illustrate how such a fundamental understanding of the way catalysts operate can provide a basis for rational design of catalysts and further technological innovations in the plastic industry.

The third article, "The Computational Challenges in Simulating Large DNA over Long Times" by Tamar Schlick, Daniel Beard, Jing Huang, Daniel Strahs, and Xiaoliang Qian, describes the computational challenges and solution strategies in simulations of the long-time dynamics of DNA, with example applications. Such simulations call for a sophisticated array of algorithms appropriate to DNA's impressive spectrum of spatial and time scales.

Lastly, "Methods in Computational Chemistry" by Siddharth Dasgupta uses two specific examples to illustrate computational chemistry's role in solving industrial problems. The first looks at a major challenge oil refineries face: the crude from different oil wells has different hydrocarbon composition, and even crude from the same well can have different composition depending on its remaining useful life. However, each refinery must produce specific target products; quantum chemistry has provided valuable answers to these challenges. The next example addresses issues underlying the mechanism by which a polymeric film prevents oxygen from diffusing through it and thus avoids oxidative damage to food. This example links molecular dynamics and mesoscale simulation. 
W

e hope this issue gives a flavor of both the kind of accomplishments that computational chemists are proud of and the challenges that excite and stimulate us. st

\section{References}

1. D.G. Truhlar and K. Morokuma, Transition State Modeling for Catalysis, American Chemical Soc., Washington, DC,1999.

2. A.L. Parrill and M.R. Reddy, Rational Drug Design, American Chemical Soc., New York, 1999.

3. D.G. Truhlar, W.J. Howe, A.J. Hopfinger, J. Blaney, and R.A. Dammkoehler, Rational Drug Design, Springer-Verlag, New York, 1999.

4. V.V. Bulatov, T.D. de la Rubia, R. Phillips, E. Kaxiras, and N Choniem, Multiscale Modeling of Materials, Materials Research Soc., Warrendale, Pa., 1999

5. A.F. Voter, "Hyperdynamics: Accelerated Molecular Dynamics of Infrequent Events," Physical Rev. Letters, Vol. 78, No. 20, May 1997, pp. 3908-3911.
Donald G. Truhlar is Institute of Technology Distinguished Professor of Chemistry, Chemical Physics, and Scientific Computation at the University of Minnesota, where he is also the director of the Minnesota Supercomputer Institute. His research interests focus on computational chemical dynamics. He received his PhD from the California Institute of Technology. Contact him at the Dept. of Chemistry, Univ. of Minnesota, 139 Smith Hall, 207 Pleasant St. SE, Minneapolis, MN 55455-0431; truhlar@umn.edu.

Vincent McKoy is a professor of theoretical chemistry at the California Institute of Technology. His primary interests include developing methodologies and advanced computational strategies for studying electron collisions with polyatomic gases used in the plasmaprocessing steps in semiconductor fabrication and of ultrafast pump-probe photoelectron spectroscopy of wavepackets in molecules. He received his $\mathrm{PhD}$ from Yale University. Contact him at the California Inst. of Technology, Caltech Chemistry 127-72, Pasadena, CA 91125; mckoy@its.caltech.edu.

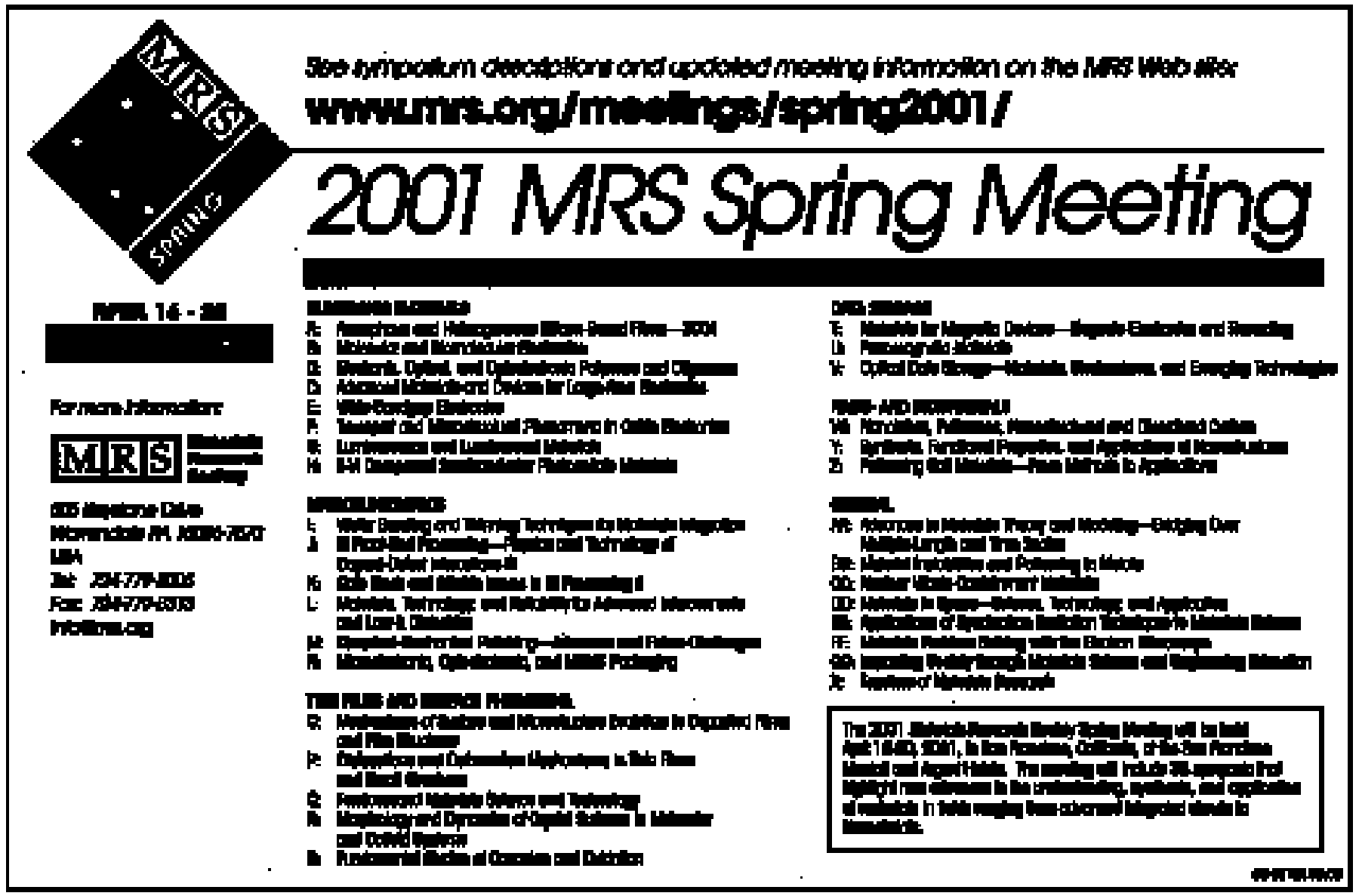

